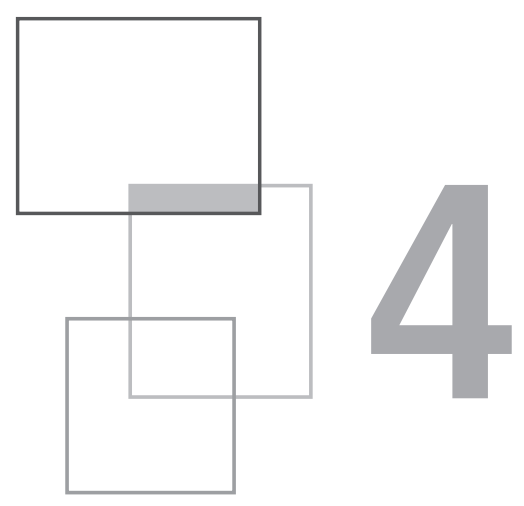

\title{
La loi sur la transition énergétique et la croissance verte (LTECV)
}

\subsection{La transition énergétique, objectifs et priorité : la France dans le contexte mondial}

La consommation d'énergie a été multipliée par dix en un siècle, accompagnant l'augmentation de la population mondiale et les développements technologiques. La contribution des énergies fossiles a crû à un rythme similaire et elles couvrent encore plus de $78 \%$ des besoins.

Cette prééminence des combustibles fossiles pose la question de leurs ressources ultimes mais également de leurs conséquences sur la pollution et l'évolution des émissions de gaz à effet de serre et du climat (voir Partie 1, Chapitre 3).

Globalement, la France est désormais quasiment non productrice de combustibles fossiles, ce qui pèse sur sa balance commerciale, très déficitaire (de 35 à 60 milliards par an selon les années).

Les émissions de $\mathrm{CO}_{2}$ de la France étaient de 333 millions de tonnes en $2016^{17}$, ce qui représente 0,91\% des émissions mondiales et 9,6\% des émissions de l'Europe des 28.

17. https://www.insee.fr/fr/statistiques/2015759 
Tableau 1 Répartition des émissions de $\mathrm{CO}_{2}$ par secteur en France en 2016.

\begin{tabular}{|c|c|c|c|c|c|c|}
\hline Secteur & Transport & Bâtiment & Industrie & $\begin{array}{c}\text { Énergie } \\
\text { + Transformation }\end{array}$ & Agriculture & TOTAL \\
\hline Millions t CO & 128 & 75 & 74 & 44 & 12 & 333 \\
\hline
\end{tabular}

Les émissions de GES (gaz à effet de serre) étaient de 454 millions de tonnes équivalent $\mathrm{CO}_{2}$. La figure ci-dessous présente l'évolution de nos émissions de 1990 à 2016 et les compare aux objectifs de Kyoto ainsi qu'aux directives européennes de 2017.

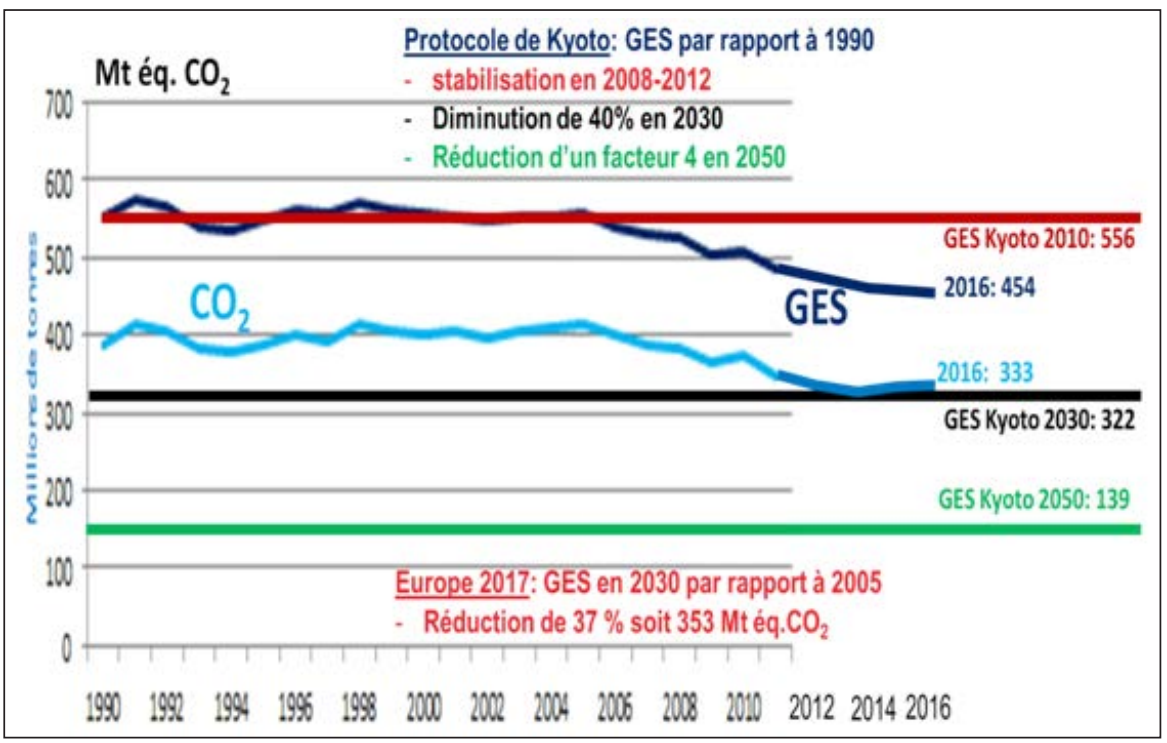

Figure 1 Émissions de GES (hors UTCF) et de $\mathrm{CO}_{2}$ en France corrigées des variations climatiques.

On constate une baisse significative des émissions à partir de 2006 puis une évolution bien moins marquée à partir de 2014 (au total 16,2\% de 1990 à 2014). Ces réductions résultent essentiellement de la baisse des émissions de $\mathrm{CO}_{2}$ du secteur manufacturier et du secteur énergie qui se sont écroulées de 42 et $44 \%$ respectivement. Les objectifs successifs du Grenelle de l'environnement et de la LTECV ont eu très peu d'impact.

Face à ces obligations et au peu de poids qu'aura la France sur l'évolution des émissions des gaz à effet de serre, il est essentiel d'examiner ce que sont les contributions proposées par d'autres pays dans le cadre des COP 21 et $22^{18}$ : sont-elles réalistes

18. http://www.diplomatie.gouv.fr/fr/politique-etrangere-de-la-france/climat/paris-2015-cop21/ les-contributions-nationales-pour-la-cop-21/article/la-carte-mondiale-des-contributions-nationales. 
et équilibrées ? Quatre pays (Chine, États-Unis, Inde et Russie) représentent à eux quatre près de la moitié des émissions mondiales. Or les contributions ${ }^{19}$ qu'ils proposent de réduction de leurs émissions de $\mathrm{CO}_{2}$, difficiles à comparer car n'ayant pas les mêmes références, montrent un déséquilibre certain. Si l'on se réfere à la même date de référence, 1990, que proposent ces 4 pays par rapport à l'Europe et la France?

- Les États-Unis (16,86 t par an et habitant ${ }^{20}:-11 \%$ en $2025^{21}$.

- La Russie (11,2 t par an par habitant) : - 25 à - $30 \%$ en 2030 mais avec une prise en compte de l'impact positif du développement de la forêt suite au réchauffement climatique.

- La Chine (7,52 t par an et habitant) : pic d'émissions en 2030 non spécifié.

- L'inde (1,73 t par an et habitant) : de - 25 à - $30 \%$ par point de PIB en 2030. La croissance du PIB étant en 2015 de 7 \% par an, leurs émissions continueront à croître fortement.

Or ces pays, comme l'Australie, grand exportateur de charbon, disposent de ressources fossiles importantes qui alimentent leurs économies. En comparaison, l'Europe indique une réduction de $40 \%$ en 2030 par rapport à 1990 pour des émissions moyennes de 6,95 t par an par habitant (France : 5,28 t). Si l'on se fie à l'ensemble de ces chiffres, le ratio entre un Américain et un Européen va continuer à croître et des pays comme l'Inde et la Chine pourraient avoir des émissions supérieures en 2030. L'effort européen pourrait donc être d'une efficacité très relative, l'impact du $\mathrm{CO}_{2}$ sur le climat étant global.

Au niveau européen, les rejets de gaz à effet de serre (GES) par personne et par État varient considérablement.

Ils sont par exemple de $35 \%$ plus élevés en Allemagne et au Danemark qu'en France, voire de $40 \%$ par rapport à la Suède. Or L'Europe demande pour 2030, par rapport à 2005, des réductions en fonction du PIB des pays, sans objectif de convergence des émissions.

Il s'ensuit que la France et la Suède auront des objectifs (- $37 \%)$ très voisins de celui de l'Allemagne $(-38 \%)^{22}$. Les écarts resteront ainsi quasi similaires entre ces pays en 2030. L'avantage ainsi offert à l'Allemagne et au Danemark est d'autant plus important qu'en basculant du charbon au gaz, ils peuvent à moindre coût réduire leurs émissions.

19. Les " contributions » sont des objectifs non contraignants que se fixent les pays, qui serviraient de référence lors des évaluations périodiques.

20. Global carbon Atlas 2016 : http://www.globalcarbonatlas.org/en/CO2-emissions

21. Contribution proposée par le président B. Obama de -26 à $-28 \%$ par rapport à 2005 alors que les émissions américaines ont crû de $15 \%$ de 1990 à 2030. Cette contribution est contestée par le président Trump.

22. Il s'agit d'objectifs hors ETS (European Trading Scheme). 


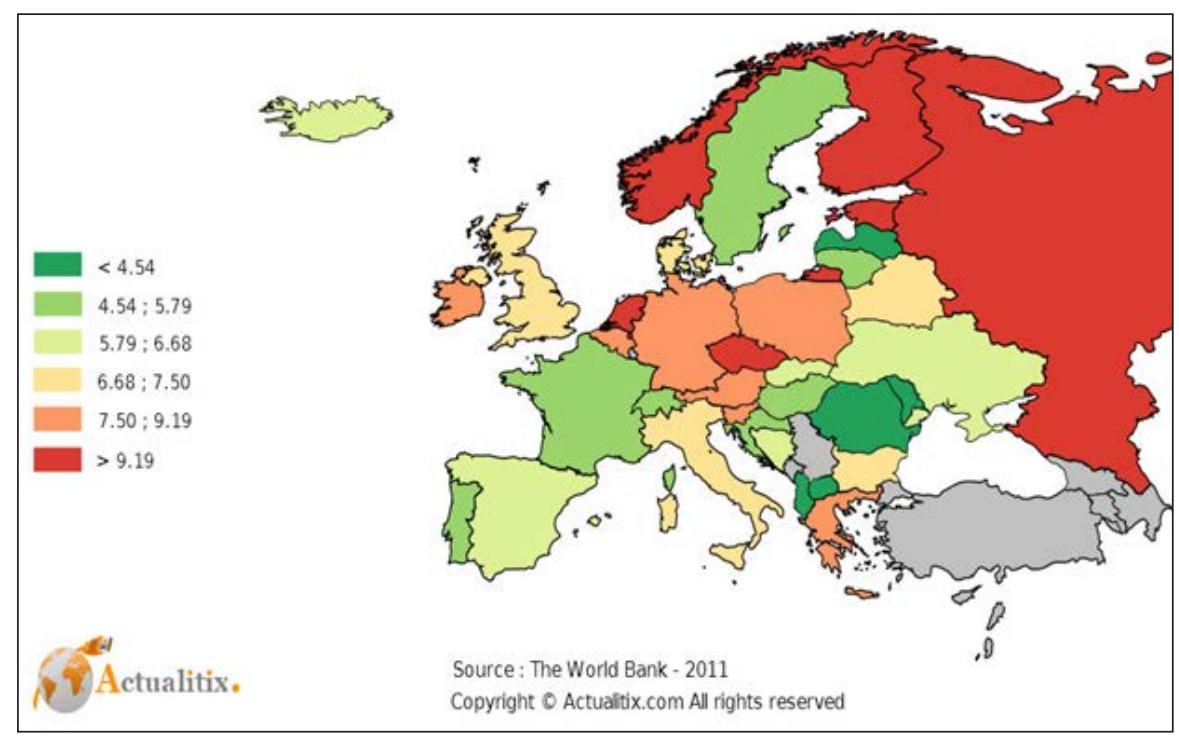

Figure 2 Émissions de GES en Europe.

L'effort européen, et a fortiori français, doit prendre en considération ce déséquilibre des contributions en tenant compte de deux évidences pour préserver leur compétitivité :

- Le $\mathrm{CO}_{2}$ a une durée de vie longue dans l'atmosphère : il y a effet cumulatif des émissions. Un million de tonnes évité dans un an sera 20 fois plus efficace qu'une tonne évitée dans 20 ans.

- Nécessité d'utiliser aux mieux les technologies les plus efficaces et les plus économiques, sans s'engager massivement sur des technologies non prouvées, chères ou peu efficaces.

\subsection{Que dit la loi sur la transition énergétique et la croissance verte (LTECV) du 17 août 2015 ?}

C'est un texte volumineux (plus de 60 pages) divisé en huit titres qui définissent chacun des objectifs dans un domaine particulier en lien avec l'énergie. L'énoncé de ces titres démontre le caractère verbeux de la loi :

Titre I : Définir les objectifs communs pour réussir la transition énergétique, renforcer l'indépendance énergétique et la compétitivité économique de la France, préserver la santé humaine et l'environnement et lutter contre le changement climatique.

Titre II : Mieux rénover les bâtiments pour économiser l'énergie, faire baisser les factures et créer des emplois. 
Titre III : Développer les transports propres pour améliorer la qualité de l'air et protéger la santé.

Titre IV : Lutter contre les gaspillages et promouvoir l'économie circulaire : de la conception des produits à leur recyclage.

Titre $V$ : Favoriser les énergies renouvelables pour diversifier nos énergies et valoriser les ressources de nos territoires.

Titre VI : Renforcer la sûreté nucléaire et l'information des citoyens.

Titre VII: Simplifier et clarifier les procédures pourgagner en efficacité et en compétitivité. Titre VIII : Donner aux citoyens, aux entreprises, aux territoires et à l'État le pouvoir d'agir ensemble.

En termes quantitatifs, les principaux objectifs fixés par la loi sont les suivants :

- Rénover énergétiquement 500000 logements par an à compter de 2017, dont au moins la moitié est occupée par des ménages aux revenus modestes, visant ainsi une baisse de $15 \%$ de la précarité énergétique d'ici 2020.

- Réduire les émissions de gaz à effet de serre de $40 \%$ entre 1990 et 2030 et diviser par quatre les émissions de gaz à effet de serre entre 1990 et 2050 (facteur 4).

- Réduire la consommation énergétique finale de $50 \%$ en 2050 par rapport à la référence 2012 en visant un objectif intermédiaire de $20 \%$ en 2030.

- Réduire la consommation énergétique primaire d'énergies fossiles de $30 \%$ en 2030 par rapport à la référence 2012.

- Porter la part des énergies renouvelables à $23 \%$ de la consommation finale brute d'énergie en 2020 et à $32 \%$ de la consommation finale brute d'énergie en 2030.

- Réduire la part du nucléaire dans la production d'électricité à $50 \%$ à l'horizon 2025 (contre $75 \%$ ).

- Atteindre un niveau de performance énergétique conforme aux normes "bâtiment basse consommation " pour l'ensemble du parc de logements à 2050.

- Réduire de $50 \%$ la quantité de déchets mis en décharge à l'horizon 2025 et découpler progressivement la croissance économique et la consommation matières premières.

La loi fixe également une limite de puissance du parc nucléaire français à 63,2 GW. Cette limitation a été introduite pour obliger EDF à arrêter la production de certaines de ses centrales de puissance (en pensant évidemment à Fessenheim) dès la mise en service de l'EPR de Flamanville.

À trop contraindre tous les domaines, la France n'établit en fait aucune réelle priorité d'action en faveur de la lutte contre le réchauffement climatique : on mêle des objectifs (climatiques par exemple) et des moyens (développement des ENR, réduction du nucléaire) qui peuvent contredire l'objectif principal de limitation des émissions de GES. La comparaison avec la situation allemande est pleine d'enseignements qui n'ont pas été pris en compte (voir Annexe). 


\subsection{Notre pays face à la LTECV}

La France, alors qu'elle est plus performante en termes de GES que l'essentiel des autres pays européens, est en retard dans les objectifs de développement des énergies renouvelables qu'elle s'est elle-même imposés pour des raisons idéologiques : à trop promettre depuis 2006 (Grenelle de l'environnement puis loi LTECV), elle se met encore une fois dans une situation diplomatique difficile. L'objectif de $23 \%$ d'EnR en 2020 est inatteignable. Pourquoi tant promettre, sans bénéfice GES évident, alors que l'Allemagne s'était limitée à $18 \%$ (qu'elle n'atteindra pas) ?

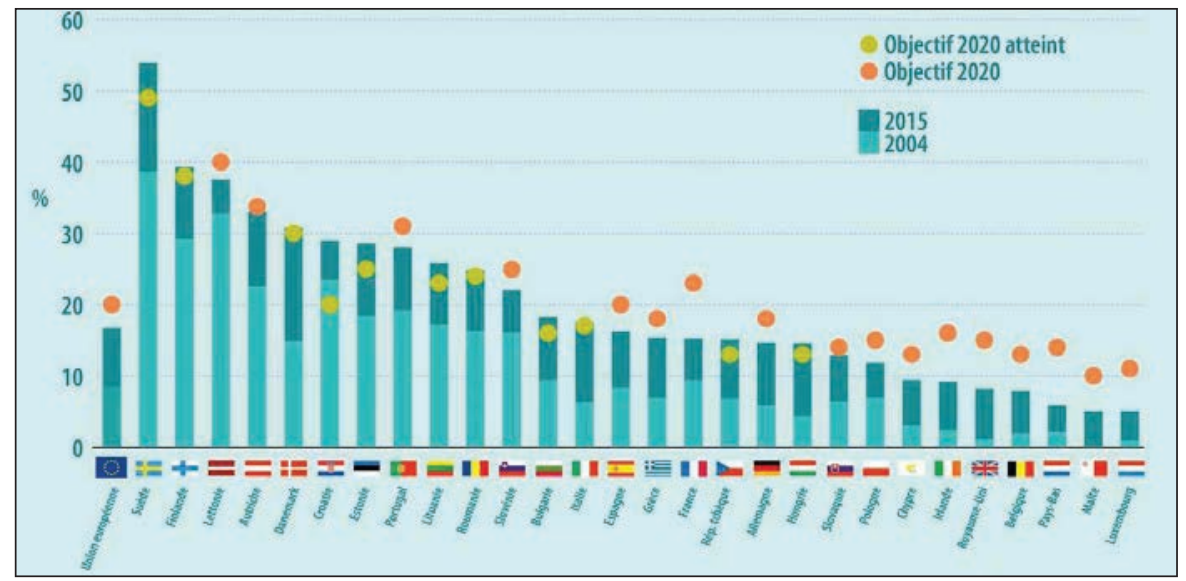

Figure 3 Part de l'énergie provenant des sources renouvelables dans les pays de l'UE en 2015 (Eurostat).

On comprend mieux cette erreur stratégique quand on observe ses émissions de $\mathrm{CO}_{2}$, soit 313,1 millions de tonnes en 2016 (source CGDD avril 2018).

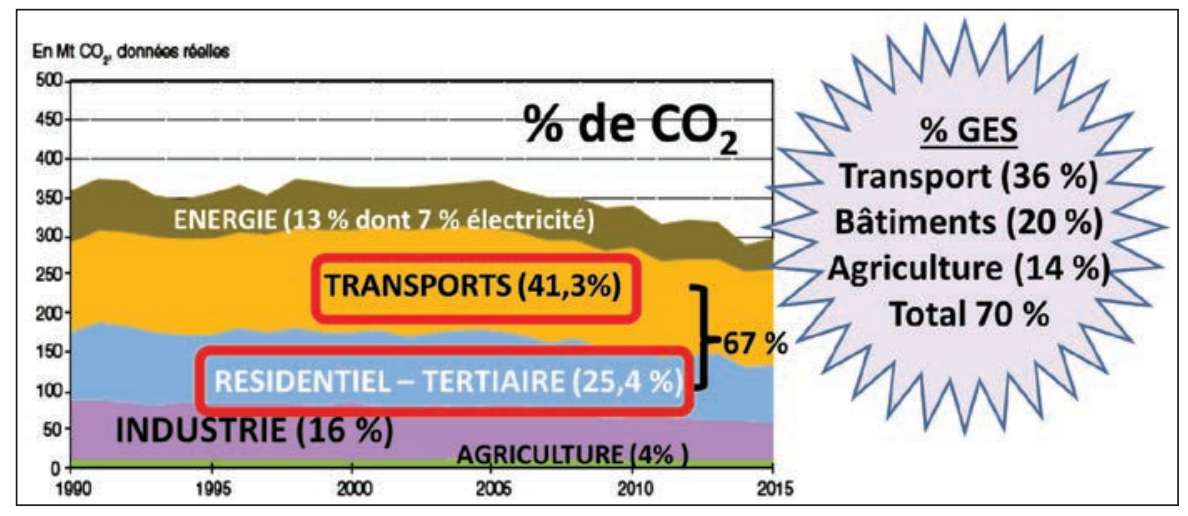

Figure 4 Les émissions de $\mathrm{CO}_{2}$ en France par secteurs et les émissions de GES en 2015. 
Il est clair que les émissions de GES proviennent essentiellement de trois secteurs, les transports, les bâtiments et l'agriculture. Or si on examine la consommation en énergie finale, seule révélatrice des progrès en efficacité énergétique, on constate des progrès très insuffisants depuis le Grenelle de l'environnement, qu'il s'agisse du niveau d'appel aux combustibles fossiles ou des émissions de $\mathrm{CO}_{2}$.

Tableau 2 Consommation d'énergie finale en France de 1990 à 2015 par secteurs et émissions de $\mathrm{CO}_{2}$ (CGDD avril 2018) ${ }^{23}$.

\begin{tabular}{|l|r|r|r|r|r|r|r|r|r|r|r|r|}
\hline \multicolumn{1}{|c|}{ Mtep } & $\mathbf{1 9 9 0}$ & $\mathbf{2 0 0 5}$ & $\mathbf{2 0 0 8}$ & $\mathbf{2 0 0 9}$ & $\mathbf{2 0 1 0}$ & $\mathbf{2 0 1 1}$ & $\mathbf{2 0 1 2}$ & $\mathbf{2 0 1 3}$ & $\mathbf{2 0 1 4}$ & $\mathbf{2 0 1 5}$ & $\mathbf{2 0 1 6}$ & $\mathbf{2 0 1 7}$ \\
\hline Consommation & 140,2 & 164,9 & 175,2 & 156,4 & 154,7 & 157,1 & 155,9 & 153,3 & 155,0 & 155,0 & 153,4 & 155,4 \\
\hline Résidentiel/tertiaire & 55,6 & 63,9 & 66,9 & 67,6 & 65,1 & 67,1 & 67,2 & 67,2 & 66,5 & 66,7 & 66,8 & \\
\hline Transports & 34,5 & 44,4 & 43,9 & 43,5 & 43,6 & 43,5 & 43,4 & 43,1 & 43,4 & 43,8 & 43,8 & \\
\hline Industrie & 30,6 & 32,4 & 31,8 & 26,5 & 27,6 & 27,8 & 26,9 & 26,7 & 26,3 & 26,2 & 26,0 & \\
\hline Emissions $\mathrm{CO}_{2}$ & 359 & 386,5 & 365,2 & 345,4 & 352,0 & 325,6 & 330,0 & 329,6 & 298,3 & 303,3 & 303,9 & 313,1 \\
\hline
\end{tabular}

Il est particulièrement frappant de constater que dans les deux secteurs essentiels du bâtiment et du transport les progrès sont nuls depuis quelques années, les niveaux étant encore supérieurs à ceux de 1990 et similaires à ceux de 2005.

La contribution des énergies renouvelables, prioritaire dans la LTECV a crû de 2005 à 2015 de 15,4 à 22,7 millions de tep, soit une augmentation de $32 \%$, mais leur part dans la consommation d'énergie finale du pays est de 4,5\% seulement. La majeure part résulte de la croissance des biocarburants (41\%), alors que ceuxci sont peu efficaces du point de vue des émissions de $\mathrm{CO}_{2}$, et de la biomasse/ déchets (39\%). Le développement des énergies renouvelables intermittentes, éolien et solaire, a contribué pour $18 \%$ seulement à cette croissance.

\subsubsection{Les résultats de la LTECV par secteurs}

\subsubsection{L'électricité}

L'apport d'énergie des énergies renouvelables intermittentes, solaire et éolien, reste faible malgré des conditions financières offertes très généreuses ${ }^{24}: 2,2$ millions de

23. Difficile de trouver des chiffres cohérents : émissions 2016 de $\mathrm{CO}_{2}$ étant évaluées entre 293 et $333 \mathrm{Mt}$ selon les sources publiques.

Chiffres clés du climat 2017/2018 : 328 Mt de $\mathrm{CO}_{2}$ en 2015 et 457,1 Mt eq. $\mathrm{CO}_{2}$ de GES hors $\operatorname{UCTF}\left(74 \% \mathrm{CO}_{2}\right)$.

24. L'économie de ces énergies repose sur une obligation d'enlèvement de l'énergie produite imposée au réseau et sur des tarifs publics offrant une très forte rentabilité sur fonds propres : http://www.cre.fr/documents/publications/rapports-thematiques/couts-et-rentabilitedes-enr-en-france-metropolitaine 
tep et 1,36\% de notre énergie finale en 2016. La baisse correspondante d'émissions de $\mathrm{CO}_{2}$ a été au mieux de 6 à 10 millions de tonnes par an sur un total initial d'environ 350. Or ce gain coûtera à la collectivité 5,48 milliards en 2018 d'après la Commission de régulation de l'électricité $(\mathrm{CRE})^{25}$, soit un coût de la tonne de $\mathrm{CO}_{2}$ évité de $610 €^{26}$.

Le secteur électrique est encadré par la loi mais également dans un plus grand détail par la Programmation pluriannuelle de l'énergie (PPE). Un élément très médiatisé de la loi sur la transition énergétique (LTE) est l'engagement de réduire d'un tiers, à $50 \%$, la contribution de l'énergie nucléaire à la production d'électricité à l'horizon 2025, en développant les énergies renouvelables électrogènes : cette orientation figure clairement dans la PPE, associée à un objectif de $40 \%$ d'EnR en 2030 et à une limitation à 63,2 GW, niveau actuel, de la puissance nucléaire installée.

En novembre 2017, Nicolas Hulot a reconnu que cet objectif était irréalisable et a indiqué qu'il pourrait, dans une révision de la PPE, être reporté à 2030 ou 2035. Cet objectif serait probablement tout aussi irréaliste si les objectifs de réduction des GES étaient maintenus.

En Allemagne, à titre de comparaison, avec un parc éolien + solaire électrogène de 90 GW en 2016, supérieur de $43 \%$ en puissance à notre parc nucléaire, le secteur électrique a émis $477 \mathrm{~g} / \mathrm{kWh}$ car ces EnR n'ont produit que $20 \%$ de l'électricité (les fossiles en produisaient 52,6\% : voir l'annexe).

Le Gouvernement français, dans son arrêté relatif à la PPE 2018/2023 ${ }^{27}$, propose d'accélérer encore le processus de déploiement des électricités éolienne et photovoltaïque. Que spécifie la PPE pour 2023 :

- par rapport à 2015, l'éolien terrestre devrait passer de 9120 à 21800 ou $26000 \mathrm{MW}$ selon les deux scénarios haut et bas présentés, avec un rythme de raccordement au réseau qui va passer de $1200 \mathrm{MW} / \mathrm{an}$ à 1500 ou $1800 \mathrm{MW} / \mathrm{an}$;

- l'objectif visé pour l'éolien marin est de $3000 \mathrm{MW}$;

- le solaire devrait voir son rythme de raccordement augmenter de $900 \mathrm{MW} / \mathrm{an}$ à 1600 ou $2000 \mathrm{MW} / \mathrm{an}$ et la puissance installée de $6200 \mathrm{MW}$ à 18200 ou $20200 \mathrm{MW}$.

Ces énergies présentent bien sûr l'avantage d'être renouvelables, mais leur intermittence et leurs productions aléatoires les rendent dépendantes de moyens de centrales de back-up, qui sont majoritairement en France des centrales nucléaires et hydroélectriques non émettrices de gaz à effet de serre (GES) et, dans une moindre mesure, des centrales à combustibles fossiles. Au total, l'électricité intermittente devrait évoluer selon les hypothèses haute ou basse de $16500 \mathrm{MW}$ à 43000 ou $49200 \mathrm{MW}$, soit environ $70 \%$ de la puissance actuelle des centrales nucléaires. L'impact de ce

25. http://www.cre.fr/documents/deliberations/decision/cspe-2018/consulter-la-deliberation

26. Le prix de la tonne de $\mathrm{CO}_{2}$ sur le marché des quota européens est inférieur à $10 €$ par tonne et le gouvernement projette un coût plancher de $30 € / \mathrm{t}$ seulement en 2030 .

27. https://www.legifrance.gouv.fr/eli/decret/2016/10/27/DEVR1619015D/jo/texte. 
programme a été évalué ${ }^{28}$ en s'appuyant sur la réalité de la productivité de ces énergies en France. Le résultat est le suivant pour le seul scénario bas, le scénario haut étant encore plus irréaliste (voir tableau 3).

Tableau 3 Contribution des énergies éolienne et solaire à la production d'électricité en 2023 : scénario bas de la PPE.

\begin{tabular}{|l|c|c|}
\hline & Éolien + solaire & $\begin{array}{c}\text { Scénario bas 2023 } \\
\text { Autres énergies }\end{array}$ \\
\hline Production TWh & 72,1 & 420 \\
\hline \% contribution à la production annuelle & 14,7 & 53,3 \\
\hline Efficacité de production & 19,1 & \\
\hline Puissance maxi MW & 25166 & 89171 \\
\hline Puissance mini MW & 370 & 13947 \\
\hline
\end{tabular}

L'impact de ce développement peut être mieux compris en examinant la figure ci-dessous qui donne une simulation des productions d'électricités intermittentes basée sur un climat annuel moyen (2013) et les puissances installées prévues par la PPE.

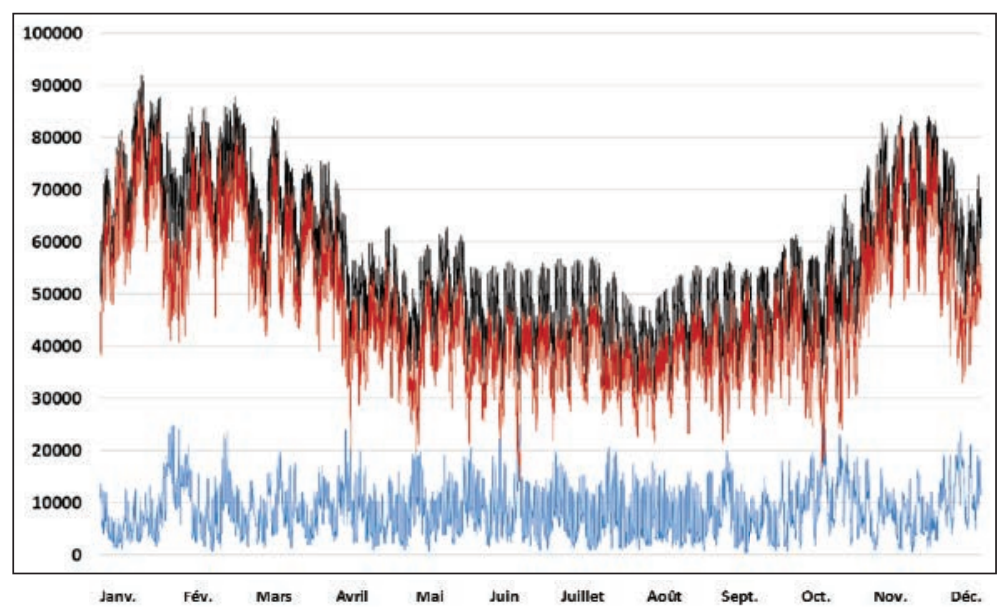

Figure 4 Productions horaires 2023 en MWh (scénario bas du projet d'arrêté) dans I'hypothèse de conditions climatiques identiques à celles de 2013. Les productions de l'éolien et du solaire en 2013 sont amplifiées en se projetant en 2023 au prorata des puissances installées : en noir, la production totale ; en bleu, « éolien + solaire » (43 GW de puissance installée); et en rouge, l'ensemble des autres moyens de production en 2023.

28. https://www.sauvonsleclimat.org/images/articles/pdf_files/etudes/Analyse $\% 20$ projet $\% 20$ arrete\%20PPE\%202018_2023.pdf 
Le tableau ci-dessus montre que la contribution intermittente restera faible (15\%) malgré une capacité portée à $43 \mathrm{GW}$ (le nucléaire produit $75 \%$ de l'électricité avec une capacité de $63 \mathrm{GW}$ ). Le graphique pour sa part montre que sa variabilité au pas horaire est considérable (courbe bleue), été comme hiver, et que les périodes de faibles productions éolienne et solaire, l'hiver en particulier, imposent de conserver une puissance pilotable (courbe rouge : nucléaire, hydraulique, fossile) quasiment identique à l'actuelle et répondant à la quasi-totalité de la consommation (courbe noire). Dans ce scénario, la puissance installée totale passe de 129,3 GW en 2015 à $151 \mathrm{GW}$ en $2013^{29}$. La puissance intermittente est essentiellement ajoutée et ne remplace que très partiellement la puissance pilotable : c'est un investissement qui s'ajoute.

L'investissement de 2016 à 2023 pour produire 43 TWh par an de plus en 2023 qu'en 2015 (soit au total 72 TWh par an en 2023), sera d'environ 50 à 60 milliards et le gain $\mathrm{CO}_{2}$ nul ou négatif selon le scénario d'arrêt de centrales nucléaires (un chiffre maximum de 17 tranches a été cité en juillet 2017 par le ministre). Il faudrait en effet compenser ces arrêts par de nouvelles centrales à gaz. L'équipement (nacelles éoliennes, panneaux solaires) est importé à près de $100 \%$, ce qui représente, suivant les technologies, de 50 à $70 \%$ de l'investissement total.

Les productions éolienne et solaire sont souvent de même ampleur dans l'ensemble de l'Europe de l'Ouest, soumises aux influences atlantique et sibérienne ${ }^{30}$. L'espoir d'un foisonnement favorable des productions nationales est illusoire ${ }^{31}$. De cette simultanéité de productions fortes ou insignifiantes résulte une fragilisation considérable du secteur européen de l'électricité, les moyens de production lourdement subventionnés que sont le solaire et l'éolien ayant capté l'investissement et les moyens pilotables, pourtant essentiels, étant confrontés à un écroulement du marché. Ils ne sont plus renouvelés, voire arrêtés sauf subventionnés par un mécanisme de capacité (voir Partie 2, chapitre 3).

L'introduction d'électricité intermittente a d'autre part des limites physiques, faute de disponibilité de moyens de stockage, limites liées à la nécessité d'équilibrer en permanence le réseau. Cette question, très complexe, a été étudiée en détail par $\mathrm{EDF}$, avec prise en compte de l'équilibre instantané du réseau français et du réseau européen au pas horaire. Cette étude confirme que l'introduction de fortes capacités de sources intermittentes :

- ne réduit que faiblement la capacité nécessaire de centrales pilotables (d'où un double investissement pour la même production) ;

29. En Allemagne, en 2016, la puissance installée est de proche de $200 \mathrm{GW}$ pour une puissance appelée maximale de $80 \mathrm{GW}$ seulement. La puissance des centrales fossiles est encore de $76 \mathrm{GW}$, avec un nucléaire à 10,8 GW.

30. https://www.sauvonsleclimat.org/images/articles/pdf_files/etudes/A\%20Eolien\%20 en\%20 Europe,\%20foisonnement\%20et\%20production\%20de\%20H2.pdf

31. https://www.sauvonsleclimat.org/images/articles/pdf_files/etudes/131120_Flocard_ FoisonnementEolien.pdf:A\%20Eolien\%20en\%20Europe, $\% 20$ foisonnement $\% 20 \mathrm{et} \% 20$ production $\% 20 \mathrm{de} \% 20 \mathrm{H} 2$.pdf 
- ne peut dépasser $40 \%$ de la production annuelle en Europe sans risquer de fragiliser le réseau, à condition de respecter des taux instantanés d'électricité intermittente variant de $25 \%$ pour des demandes faibles, à $35 \%$ pour des demandes moyennes et à $70 \%$ lors des pics de consommation ;

- rendrait nécessaire la maîtrise de l'écrêtage de la production intermittente et la mise en œuvre d'un stockage/déstockage d'électricité massif qui n'est techniquement qu'hypothétique.

Lélectricité intermittente est principalement injectée dans le réseau au niveau des réseaux moyenne et basse tension d'ENEDIS et non dans le réseau haute tension de RTE. Or, si ce dernier est maillé et permet de distribuer le courant dans toutes les directions (le soleil est au sud et l'éolien surtout au nord), le second est étoilé et donc conçu pour véhiculer le courant dans un seul sens. Les productions solaires et éoliennes injectées dans le réseau ENEDIS doivent être en partie notable refoulées vers le réseau RTE faute d'usagers locaux : c'est donc une refonte importante des réseaux qui doit être engagée pour accompagner la croissance des énergies intermittentes.

La baisse des émissions de $\mathrm{CO}_{2}$ du secteur électrique est au mieux actuellement de 8 à 10 millions de tonnes par an sur un total initial d'environ 35. Ce gain coûtera à la collectivité 5,48 milliards d'euros en 2018 via la Contribution au service public de l'électricité (CSPE) d'après la Commission de régulation de l'électricité (CRE) ${ }^{32}$, soit un coût de la tonne de $\mathrm{CO}_{2}$ évité de $610 €$.

Avec un investissement inférieur, 55 milliards d'euros, le parc nucléaire actuel serait prolongé de 20 ans et produirait 417 TWh par an, soit un investissement par TWh 5,8 fois inférieur. Or ce sont des énergies comparables, en ce sens qu'elles réclament de forts investissements, bénéficient de coûts d'exploitation modérés et émettent très peu de GES.

La France dispose d'un investissement considérable, largement amorti, le nucléaire. Pour progresser vite dans la décarbonation, elle doit sappuyer sur cet atout en développant les usages électriques dans tous les domaines. Brider la production comme le prévoit la LTCEV est une faute vis-à-vis du risque climatique et une catastrophe du point de vue économique car enchérissant le coût de l'électricité (voir la situation allemande avec une électricité deux fois plus coûteuse) et mobilisant d'énormes capitaux dans les renouvelables avec une efficacité négligeable vis-à-vis de la décarbonation. Le résultat de la LTECV va être une accentuation de la précarité énergétique qui touche déjà 4 millions de foyers : le Gouvernement a dî mettre en place un chèque énergie pour ces foyers en 2016, de 144 à $227 €$ par an.

32. http://www.cre.fr/documents/deliberations/decision/cspe-2018/consulter-la-deliberation 


\subsubsection{Le secteur du bâtiment}

Il représente plus du quart des émissions de $\mathrm{CO}_{2}$ du pays, et souffre d'un parc de logements et de bâtiments tertiaires pour moitié anciens, antérieurs à 1975. La LTECV fixe des objectifs ambitieux de rénovation de 500000 logements par an pour les amener à une consommation de $150 \mathrm{kWh} / \mathrm{m}^{2}$ par an. La réalité en est éloignée avec des rénovations annuelles inférieures de $40 \%$ et surtout des niveaux de performance énergétique atteints très limités et très mal évalués. En ce qui concerne les bâtiments neufs, ils ne doivent pas dépasser $50 \mathrm{kWh} / \mathrm{m}^{2} /$ an depuis 2012 (bâtiments basse consommation). Mais leur impact sur les émissions sera faible car ce sont souvent des surfaces ajoutées et le volume annuel réalisé n'est que d'environ $1 \%$ du parc actuel.

La dimension du problème est considérable avec 34 millions de logements qui émettent en moyenne $240 \mathrm{kWh}$ par $\mathrm{m}^{2}$ et par an. Le chauffage fossile perdure encore dans $57 \%$ du parc en 2014 ( $43 \%$ gaz et $14 \%$ fuel). Il faut ajouter de plus près d'un milliard de $\mathrm{m}^{2}$ dans le tertiaire. Mais la règle technique qui s'impose aux constructions neuves depuis 2012 (RT 2012) a privilégié le gaz dans le bâti neuf, aux dépens d'un chauffage électrique performant (pompes à chaleur et cumulus électriques) ${ }^{33}$, l'objectif étant de réduire la production d'électricité nucléaire.

- Le coût de l'amélioration de l'efficacité énergétique dans les logements sera considérable, environ $30000 €$ par logement ancien (pour 18,6 millions construits avant 1975), pour réduire leur consommation de $50 \%$ : soit un total d'environ 450 milliards $€$ pour les 15 millions de logements les moins performants. C'est du long terme!

- L'utilisation de la biomasse chaleur pourrait être renforcée. Elle fournit aujourd'hui environ 5,6 \% de notre énergie finale. Elle pourrait croître de 15 à $20 \%$ en 10 ans mais sera en compétition avec les autres usages, dont les constructions bois ${ }^{34}$. Ce ne sera possible que par une réorganisation drastique du secteur car il est très éclaté (3,3 millions de propriétaires pour $75 \%$ de la forêt), donc peu rentable et faisant appel à des technologies vieillissantes. L'exploitation de la forêt est de plus bridée par une organisation «à la française » avec cinq ministères et dix interprofessions (source Cour des comptes).

- Le biogaz chaleur ${ }^{35}$ a également un potentiel en France, mais est encore peu développé bien que la technologie soit mature. Il ne représente que 0,13 million de tep en 2015, soit $0,1 \%$ de notre énergie finale. L'objectif est de le doubler d'ici 2023 et de le quadrupler en 2030. La difficulté provient d'une ressource limitée, de sa dispersion sur de vastes territoires et d'une proximité ou non d'un gazoduc pour injection dans les réseaux. Il en est résulté une dérive en Allemagne, la moitié de leur production (5 à 6 Mtep) étant issue de plantations

33. En 2013 le gaz représentait : collectif $72,6 \%$, individuel groupé $55 \%$, maisons $22 \%$.

34. Construire en bois est un moyen de stocker du carbone sans l'envoyer dans l'atmosphère sous forme de $\mathrm{CO}_{2}$ en le brûlant !

35. Le biogaz électrique représente en 2015 2,44 TWh soit 0,45\% de notre électricité. 
de maïs en complément des déchets agricoles ${ }^{36}$ et urbains (l'Europe a décidé en 2017 de limiter à $30 \%$ l'apport de plantes cultivées).

- La géothermie basse température est applicable au chauffage collectif dans des zones, comme la région parisienne, qui disposent de nappes à moyenne profondeur (autour de $1000 \mathrm{~m}$ ). Elle ne peut s'implanter que dans des ensembles disposant déjà d'un réseau de chaleur ou dans des opérations neuves. Son déploiement, intéressant en le couplant à des pompes à chaleur, sera très progressif. La géothermie pourrait doubler mais à partir d'une contribution qui n'est aujourd'hui que de $0,13 \%$ de notre énergie finale.

Globalement, l'effort de rénovation thermique de l'habitat ancien, coûteux, ne peut être que de long terme et ne pourra réussir que grâce à une reprofessionnalisation du secteur, source d'emplois, mais qui sera longue à mettre en œuvre car dépendante de formations à moyen terme. Les renouvelables thermiques représentent une solution élégante à la décarbonation mais il ne faut pas se leurrer, les ressources, qu'il s'agisse de biogaz ou de biomasse solide, sont limitées et en compétition avec d'autres secteurs.

Il faudra réviser en urgence la RT 2012 en établissant une contrainte d'émission de $\mathrm{CO}_{2}$ par $m^{2}$ et par an et non en énergie primaire.

Il faut commencer par les rénovations les plus rentables, largement étudiées par l'Union française de l'électricité : remplacement du chauffage fuel, puis du chauffage gaz (substitution par l'électricité en périodes creuses), isolation des combles, installation de pompes à chaleur électriques et chauffe-eau solaires par exemple.

Globalement, en opposition avec les objectifs de la LTECV, il faudrait remettre l'électricité au premier rang, avec des solutions performantes déjà prouvées, une domotique adaptée aux productions s'appuyant sur les compteurs intelligents.

La production d'énergies renouvelables thermiques devrait être préférentiellement développée car plus efficace que les EnR électriques.

Le biogaz apporte aux agriculteurs un complément de ressources.

Le solaire thermique ne nécessite pas de réseau et est complémentaire du chauffage électrique.

36. Le lisier issu des porcheries est de plus inapte à fournir efficacement du méthane, d'où un ajout de mais. 


\subsubsection{La décarbonation des transports}

Les deux moyens les plus évidents sont l'électrification des véhicules et l'utilisation de biocarburants ${ }^{37}$.

Depuis 2006, l'effort a porté préférentiellement sur les seconds avec un résultat très mitigé. En effet les biocarburants de première génération (essence et diesel), développés à marche forcée de 2006 à 2010 suite au Grenelle de l'environnement, se révèlent très consommateurs de terres arables ${ }^{38}$ et ont une efficacité $\mathrm{CO}_{2}$ modeste pour un prix élevé.

En 2015, leur production était de 3 millions de tep, soit 1,8\% de notre énergie finale et 5,8 \% des carburants. Les subventions attribuées représentaient déjà 3 milliards $€$ par an et l'efficacité $\mathrm{CO}_{2}$ étant faible ${ }^{39}$, le programme a dû être très ralenti. L'Europe limite désormais leur pénétration à $6 \%$ de la consommation de carburants, niveau déjà atteint en France. On constate ici l'inconvénient d'une politique très volontariste de développement d'une source d'énergie mal évaluée au départ.

En ce qui concerne les carburants de seconde génération, ils sont encore au stade de développement et se révèlent complexes en raison de la multiplicité des coupes chimiques dans la distillation et du prix d'une étape supplémentaire dans le process. Aucun pilote technologique complet ne fonctionnerait encore. La Programmation pluriannuelle de l'énergie ne prévoit qu'une production supplémentaire de $10 \%$ en 2023, soit 0,3 million de tep sous réserve d'une levée de ces difficultés.

La généralisation de véhicules roulant avec des piles à combustible alimentées par de l'hydrogène n'est pas envisageable massivement à court terme et devra prouver sa compétitivité toutes externalités incluses, étant donné son faible rendement global $^{40}$. Elle devra aussi reposer sur une électricité réellement décarbonée.

\subsubsection{L'apport global des énergies renouvelables}

En examinant l'ensemble de celles-ci, on ne peut que constater une contribution qui restera limitée d'ici 2023.

Le respect des objectifs de la LTECV, déjà ambitieux, conduirait à une contribution à l'énergie finale des EnR thermiques qui augmenterait de $19 \%$, avec un apport supplémentaire de 3 Mtep. Ils sont à soutenir raisonnablement car généralement

37. Nous ne traiterons pas ici des évolutions sociologiques, autopartage, travail à domicile, développement des transports en commun.

38. Environ l'équivalent de 3 tep par hectare pour la betterave et de 0,9 tep par hectare pour le colza : il faut donc environ $1 / 3$ d'hectare pour une voiture à essence faisant $20000 \mathrm{~km} / \mathrm{an}$ et 1 ha pour une voiture diésel (pour des biocarburants à $100 \%$ ).

39. Le gain réel d'émissions de $\mathrm{CO}_{2}$ par rapport à l'essence est d'environ $41 \%$ pour la betterave et $15 \%$ pour le blé (à proscrire !) et pour le diésel de $43 \%$ pour le colza.

40. Rendement électrolyse $0,75 \%$, rendement PAC $50 \%$, soit rendement global $37 \%$. Si électrolyse à partir d'éolien + solaire avec une productivité de $20 \%$, le rendement global n'est plus que de $7,5 \%$. 
assez efficaces, créateurs d'emplois nationaux (sauf pour l'éolien et le solaire), et représentent des compléments utiles de revenus pour les agriculteurs.

Tableau 4 Proportion des énergies renouvelables thermiques entre 2016 et 2023 (en Mtep absolus et en pourcentage de l'énergie finale).

\begin{tabular}{|l|r|r|}
\hline \multicolumn{1}{|c|}{$\begin{array}{c}\text { Energies renouvelables thermiques } \\
\text { (hors applications non énergétiques) }\end{array}$} & \multicolumn{1}{|c|}{2016} \\
\hline $\begin{array}{l}\text { BIOMASSE SOLIDE dont déchets (renouvelables } \\
\text { 1,1 et non renouvelables 1,2) }\end{array}$ & 11,7 & 13,5 \\
\hline BIOGAZ & 0,76 & 0,8 \\
\hline SOLAIRE THERMIQUE & 0,11 & 0,34 \\
\hline BIOCARBURANTS & 2,35 & 3,3 \\
\hline GEOTHERMIE & 0,24 & 0,45 \\
Pompes à chaleur & 2,18 & 3 \\
\hline TOTAL EnR Thermiques Mtep & 17,3 & 21,4 \\
Pourcentage énergie finale & $(11,3 \%)$ & $(14 \%)$ \\
\hline
\end{tabular}

\subsection{Notre pays face à la LTECV dans le domaine électrique}

Paradoxalement, le consommateur d'électricité ne voit pas la baisse des prix de marché de l'électricité sur sa facture ; au contraire, elle s'envole depuis quelques années. D'environ $130 € / M W h$ TTC il y a dix ans (tarif bleu $6 \mathrm{kVA}$ ), elle est aujourd'hui de $175 € / \mathrm{MWh}$, soit une augmentation de $35 \%$ (quand l'indice des prix n'a progressé que de $15 \%$ sur la même période). Ce prix se décompose en $36 \%$ pour l'énergie, $29 \%$ pour l'acheminement et $35 \%$ de taxes constituées à plus d'un tiers par la Contribution au service public de l'électricité (CSPE, couplée depuis le $1^{\mathrm{er}}$ janvier 2016 avec la Taxe intérieure sur la consommation finale d'électricité, TICFE).

Les investissements dans les énergies intermittentes sont considérables : 1,2 à 1,5 milliard par GW pour l'éolien terrestre, 4 à 5 milliards par GW pour l'éolien marin (avec un coût d'exploitation très supérieur, du double au triple) et, selon le type d'installation de 1 à 4 milliards par GW pour le solaire. Doivent être pris en compte également le développement des réseaux et les moyens de secours en cas de pénurie de vent et d'ensoleillement (réserves de capacité ou stockage).

Entre 2004 et 2016, la CSPE a été multipliée par cinq, passant de 4,5 €/MWh à $22,5 € / \mathrm{MWh}$, soit huit milliards d'euros en 2017. À titre de comparaison, les dépenses de maintenance et d'exploitation (y compris d'achat de combustible) de tout le parc nucléaire français seront de l'ordre de six milliards d'euros par an sur la 
période 2014-2030 selon la Cour des Comptes alors que le nucléaire fournit huit fois plus d'électricité que les énergies renouvelables et n'est pas subventionné par des tarifs d'achat.

Cette hausse passée de la CSPE est liée principalement aux tarifs d'achat éolien et photovoltaïque (pour plus de 5,6 milliards d'euros par an à partir de 2017). Cette augmentation n'a cependant pas permis d'absorber totalement les charges liées aux subventions des EnR : l'État a donc accumulé fin 2015 une dette de près de six milliards d'euros par an auprès d'EDF, qui fait désormais l'objet d'un échéancier de remboursement jusqu'en 2020. Si on peut espérer que, grâce à des gains de compétitivité, les nouveaux projets de solaire alourdiront moins la CSPE, ce ne sera probablement pas le cas de l'éolien ${ }^{41}$, et du coût de transport de l'électricité en raison des besoins de développement des réseaux HT pour les parcs importants ou MT et BT pour la distribution du solaire et de l'éolien diffus ${ }^{42}$.

D'ici 2023, la PPE adoptée en 2016 pourrait conduire à un doublement de ce soutien financier aux EnR (principalement pour le solaire et l'éolien offshore, très coûteux. En Allemagne, ce soutien aux EnR atteint près de 23 milliards d'euros par an en 2016 (voir en annexe la situation actuelle en Allemagne).

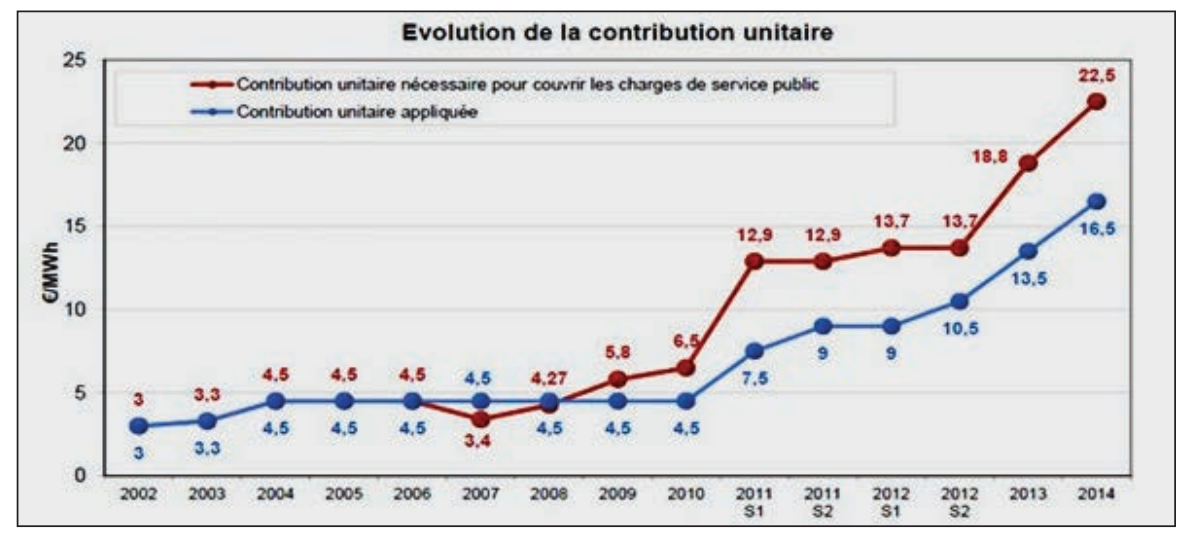

Figure 5 Évolution de la contribution unitaire de la CSPE en $€ / M W h$ telle qu'elle apparaît sur la facture EDF (courbe bleue) et telle qu'elle devrait être pour couvrir toutes les charges imposées à EDF.

41. Il faudra en effet payer pendant 20 ans un tarif de $220 € / M W h$ l'éolien marin ( $3 \mathrm{GW})$, soit 6 fois le prix du marché, et juste avant son départ, Madame Royal a réduit de 82 à $72 € / \mathrm{MWh}$ le prix de l'éolien terrestre (pour moins de $6 \mathrm{MW}$ ) mais a porté de 15 à 20 ans l'application de ce prix.

42. Il faut prévoir environ un milliard par an pour chacun des deux réseaux, soit environ 14 milliards $€$ d'ici 2023. L'essentiel des productions de l'éolien et du solaire est injecté dans le réseau ENEDIS (BT et MT), plus de la moitié devant être refoulée vers le réseau HT de RTE pour le distribuer à longue distance. 


\subsection{Conclusions}

\subsubsection{Des objectifs contradictoires}

La LTECV repose ainsi sur une confusion des objectifs destinée à préserver, au moins en apparence, les engagements du Gouvernement, tout en s'efforçant de donner une image exemplaire de la France dans le cadre des COP. Alors que l'objectif essentiel est une réduction drastique des émissions de gaz à effet de serre (GES) au niveau mondial, le Gouvernement français continue à mettre en avant le développement des énergies renouvelables (EnR) électrogènes et la réduction du nucléaire, sans gains en émissions de $\mathrm{CO}_{2}$ et aux dépens d'autres priorités comme :

- la réduction de l'usage des combustibles fossiles dans les transports et les bâtiments ;

- l'utilisation d'une production nucléaire pourtant décarbonée et très compétitive.

L'Académie des sciences a d'ailleurs rapidement pointé cette confusion des objectifs en déclarant dans son avis sur la LTECV publié en janvier 2015 :

"Pour ce qui concerne l'énergie nucléaire, le passage à $50 \%$ à l'échéance indiquée (2025) ne tient compte ni des objectifs affichés de la loi (réduction des émissions de $\mathrm{CO}_{2}$ ), ni des facteurs techniques et économiques liés à cette réduction. Cette réduction ne pourrait se faire que si des moyens de stockage de l'électricité à grande échelle devenaient disponibles, évitant le risque de déstabilisation du réseau et le recours à des centrales thermiques de compensation génératrices de $\mathrm{CO}_{2}$, pour suppléer les énergies renouvelables ".

\subsubsection{Des conséquences économiques lourdes et une fragilisation du système électrique}

Comme partout en Europe, la France a encouragé le développement des EnR, principalement l'éolien et le solaire, initialement à l'aide de mécanismes de tarifs de rachat à prix fixes garantis de l'électricité produite, pour de longues durées (15 à 25 ans). Ceci permet de soulager l'investisseur de la prise de risque et lui évite d'être soumis aux fluctuations des prix de marché.

Plus pernicieux encore, le système permet à des investisseurs sans ou avec peu de fonds propres d'emprunter à très bas taux sur les marchés financiers l'essentiel de l'investissement, et de bénéficier ainsi d'une rentabilité sur fonds propres excessive, voire scandaleuse, avec constitution de fortunes personnelles considérables sur le dos des consommateurs. Cet enrichissement a également donné un poids considérable au lobby des énergies renouvelables (Syndicat des énergies renouvelables par exemple).

Ce système a deux inconvénients majeurs : son coût, mais aussi le fait qu'il ne répond pas à une logique d'équilibre entre l'offre et la demande. On assiste donc à un effet d'éviction totale : seuls les investissements soutenus par des aides d'État, les EnR, ou imposés dans le cadre de tarifs, le transport de l'électricité, prospèrent. 
Tous les autres ont été reportés ou annulés tandis que bon nombre d'actifs thermiques existants, principalement des centrales à gaz et à charbon, ont été fermés ou mis sous cocon sauf financement additif par un mécanisme de capacités.

Comme pour la réduction de la contribution de l'énergie nucléaire, l'Académie des sciences s'est clairement prononcée sur le sujet :

"Le développement des énergies renouvelables intermittentes éolienne et photovoltä̈que devrait se faire à un rythme prudent, en tirant profit de l'expérience acquise dans d'autres pays, en anticipant les difficultés d'insertion de ces énergies dans le réseau et de leur effet sur le système électrique dans son ensemble, et en tenant compte de la nécessité de prévoir leur compensation lorsqu'elles ne sont pas disponibles, sans que cela conduise à une augmentation des émissions de $\mathrm{CO}_{2}$ ou à des importations d'énergie électrique. Les énergies renouvelables non intermittentes dérivées de la biomasse représentent une filière prometteuse quil convient de soutenir par lintensification de la recherche scientifique et technologique sur les méthodes et les procédés (physiques, chimiques, biologiques) de transformation de ces énergies en vecteurs utilisables (électricité, carburant, gaz) avec la meilleure efficacité énergétique et économique."

Plus inquiétant encore est le déclin annoncé des capacités de production pilotables en Europe dans les prochaines années. Potentiellement :

- en France, arrêt des centrales à charbon d'ici 2024 ;

- en Allemagne, arrêt de 10,8 GW nucléaire et $21 \mathrm{GW}$ de centrales à flamme d'ici 2022 ;

- sortie du nucléaire en Suisse et Belgique (dates imprécises) ;

- arrêt progressif des centrales AGR en Angleterre (2030 environ).

C'est toute la stabilité du réseau européen qui est menacée.

Les priorités de la LTECV sont contradictoires. Ayant donné priorité au développement des énergies renouvelables, les Gouvernements successifs, faute d'être en capacité de décarboner sérieusement les deux secteurs des transports et des bâtiments, ont attribué des conditions financières extrêmement avantageuses aux énergies électrogènes intermittentes (éolien et solaire en particulier). Les conséquences économiques sont supportées essentiellement par les familles et les PME par le biais de taxes déguisées en contribution. L'efficacité de l'ensemble reste faible vis-à-vis de l'enjeu climatique et un renversement des priorités simpose:

- Priorité $N^{\circ} 1$ à la réduction des GES à prix de $\mathrm{CO}_{2}$ évité minimum en priorité dans les transports et les bâtiments. Les EnR seront utiles mais ne devraient être qu'un outil de cette stratégie au meilleur cô̂t.

- Exploiter au mieux les énergies matures les plus économiques, y compris le nucléaire, en supprimant les 3 contraintes qui lui sont imposées par la LTECV : moins de $50 \%$ de la production en 2025 (au lieu de $75 \%$ actuellement), au maximum 63,5 GW de puissance installée nucléaire et arrêt de la centrale de Fessenheim (1,8 GW et 4,25 millions de tde $\mathrm{CO}_{2}$ évitées par an). 
- Réviser en urgence la RT 2012 afin de fixer dans la construction des limites en quantité de $\mathrm{CO}_{2}$ émis par $m^{2}$ et par an plutôt qu'en énergie primaire.

- Réduire le déficit de la balance commerciale en électrifiant le pays, en particulier dans les deux domaines du transport et du bâtiment tertiaire et domestique sans faire un appel massif à des technologies importées (solaire et éolien).

Une transition énergétique est nécessaire pour combattre le changement climatique mais la France, dont l'économie est fragile, doit le faire en cohérence avec ce que font les autres pays, en préservant d'une part sa compétitivité (utiliser ses atouts) et d'autre part son système social. Il faut s'interdire une amplification de la précarité énergétique, déjà constatée, et l'abandon de la péréquation (égalité de traitement des citoyens), encouragée par l'autoconsommation et la régionalisation.

\section{ANNEXE : Le retour d'expérience de l'Allemagne}

L'Allemagne s'est imposée comme le modèle d'une politique de transition énergétique. Une analyse scientifique, technique et environnementale de leurs réalisations passées ne confirme pas les performances promises. Leur production d'électricité se décline comme suit :

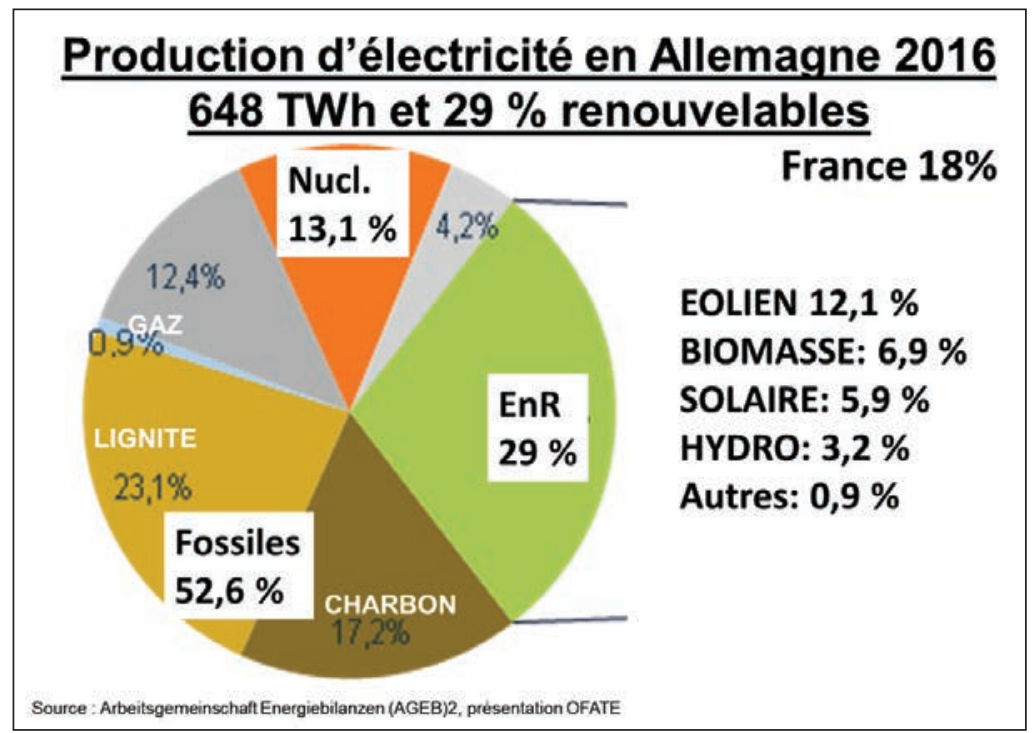

Si les énergies renouvelables représentent $29 \%$ de leur production totale, la part générée par les énergies fossiles est encore de 52,6\%, dont 40,3\% avec charbon et lignite qui sont les émetteurs majeurs de gaz à effet de serre. Les énergies intermittentes, éolien et solaire, génèrent 117 TWh par an en 2016. 


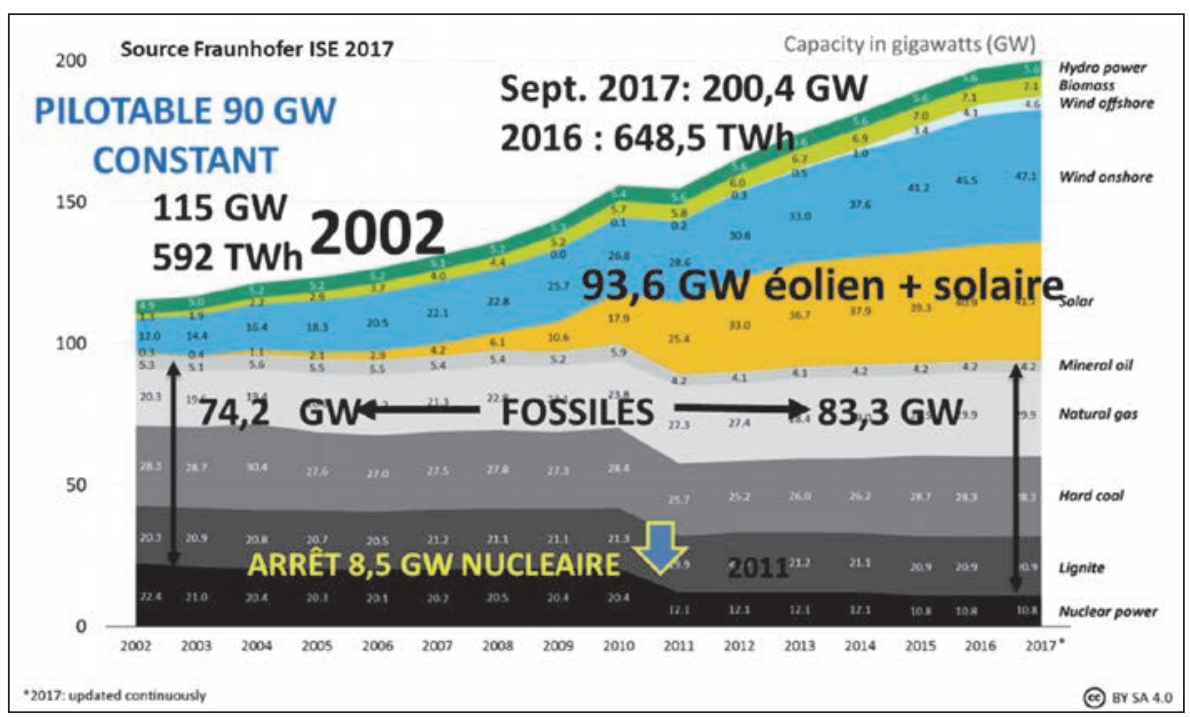

La puissance installée fossile a augmenté de $12 \%$ depuis 2002 pour compenser l'arrêt de 8,5 GW de nucléaire en 2011. La seule puissance installée du solaire et de l'éolien est de 93,6 GW alors que la puissance appelée maximale en hiver est d'environ $80 \mathrm{GW}$ seulement. On voit que $8 \mathrm{GW}$ de nucléaire arrêtés en 2011 ont été remplacés par du lignite et du gaz.

On constate un envol de la puissance installée, de $75 \%$ en 15 ans, alors que la consommation n'a évolué que de $9,5 \%$. Ceci prouve que l'essentiel de la puissance intermittente s'ajoute au parc pilotable qui sert de socle au réseau, au prix d'investissements lourds. Le prix de l'énergie intermittente ne se compare pas au prix d'une énergie pilotable car, pour une large partie, il s'ajoute à ce dernier.

L'exportation d'électricité passe de 2002 à 2016 : de - 1,5 à + 55,5 TWh. L'équivalent de $47 \%$ de la production intermittente est exporté.

Les exportations nettes se sont envolées parallèlement au développement de l'éolien et du solaire, ce qui montre que l'Allemagne n'a pas su mettre en place une gestion des centrales fossiles ayant la souplesse requise pour assurer la compensation de l'intermittence.

Quand il n'y pas de vent ni de soleil ce qui est plus fréquent qu'on ne croit, on a besoin de toute la puissance pilotable. Or l'Allemagne va arrêter 10,8 GW de nucléaire d'ici 2022 et va devenir importatrice quand il y aura peu d'intermittentes, en hiver. On voit apparaître, quand il y a trop de vent, une exportation massive avec des prix spot qui deviennent négatifs (on paye pour que l'électricité soit consommée). Les pays voisins doivent gérer des arrivées massives d'électricité imposées par l'Allemagne. Certains pays ont décidé de se protéger avec des déphaseurs qui 
bloquent l'entrée d'électricité par les réseaux interconnectés (Tchéquie, Pologne et bientôt Belgique).
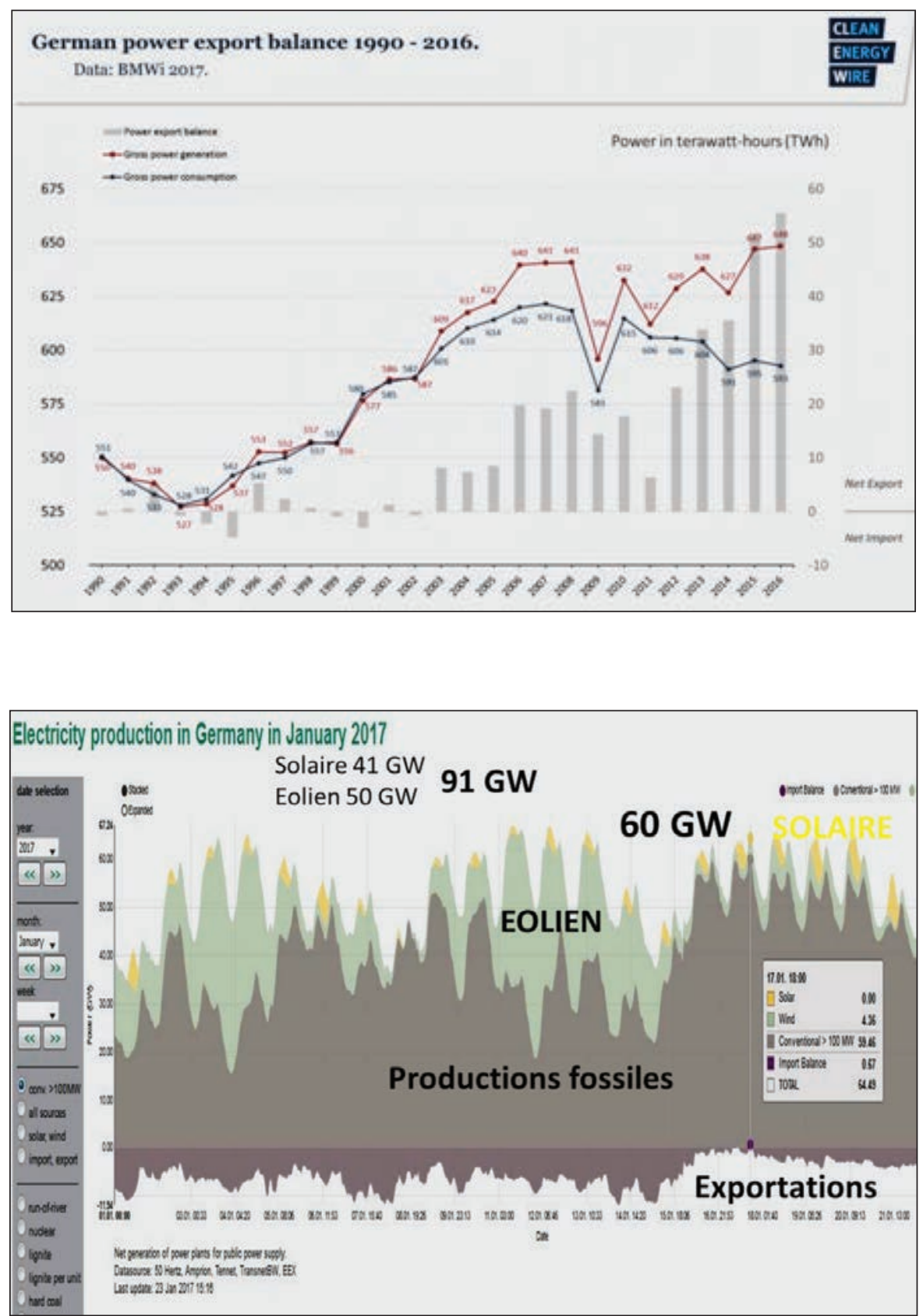
Or les Allemands vont arrêter 10,8 GW de nucléaire de plus de 2017 à 2022 et envisagent d'arrêter l'essentiel du parc charbon et lignite de 2019 à 2025 (en fonction des négociations en cours pour établir un programme de gouvernement). L'Allemagne va devenir importatrice quand il y aura peu d'intermittentes, en hiver. Celles-ci peuvent être extrêmement peu productives avec une puissance cumulée inférieure à $1 \%$ de la puissance installée.

Elles sont de plus globalement inefficaces comme le montre le tableau ci-dessous, avec une productivité par GW installé par rapport au nucléaire, dans des conditions identiques (prioritaires sur le réseau), 4,7 fois inférieure pour l'éolien et 8 fois pour le solaire en moyenne sur l'année (avec un solaire quasi absent l'hiver).

\begin{tabular}{|c|c|c|c|}
\hline SOURCE & $\begin{array}{c}\text { PUISSANCE } \\
\text { GW }\end{array}$ & $\begin{array}{c}\text { Production } \\
\text { TWh }\end{array}$ & $\begin{array}{c}\text { Production } \\
\text { par GW }\end{array}$ \\
\hline EOLIEN & 49,6 & 77,8 & 1,6 \\
\hline SOLAIRE & 40,9 & 37,5 & 0,9 \\
\hline GAZ & 29,9 & 46,4 & 1,6 \\
\hline CHARBON & 28,3 & 99,4 & 3,5 \\
\hline LIGNITE & 20,9 & 134,9 & 6,5 \\
\hline NUCLEAIRE & 10,8 & 80,0 & 7,4 \\
\hline BIOMASSE & 7,1 & 47,0 & 6,6 \\
\hline HYDRO & 5,6 & 19,1 & 3,4 \\
\hline FUEL & 4,2 & 1,0 & 0,2 \\
\hline
\end{tabular}

On voit que le lignite, large émetteur de GES, est également prioritaire, en hiver en particulier, même quand il y a du vent, car c'est un moyen de production national, sans grande souplesse, et ce sont des machines thermiques de taille considérable. Le suréquipement a eu pour conséquence l'envolée des exportations de l'Allemagne, sans que cette situation ait été discutée avec ses voisins au préalable.

La conséquence de ces investissements, 200 milliards d'euros pour le seul domaine électrogène, est nulle en ce qui concerne les émissions de gaz à effet de serre depuis 2009. De plus, comme en France, les investissements consentis sur les autres domaines (de 200 à 300 milliards d'euros selon les sources) se révèlent tout aussi peu efficaces.

On peut prévoir dans les cinq prochaines années l'impact négatif de l'arrêt de 10,8 GW de nucléaire (d'ici 2022), que l'électricité solaire et éolienne ne pourra compenser totalement. C'est tout l'Energiewende qui court à l'échec. 


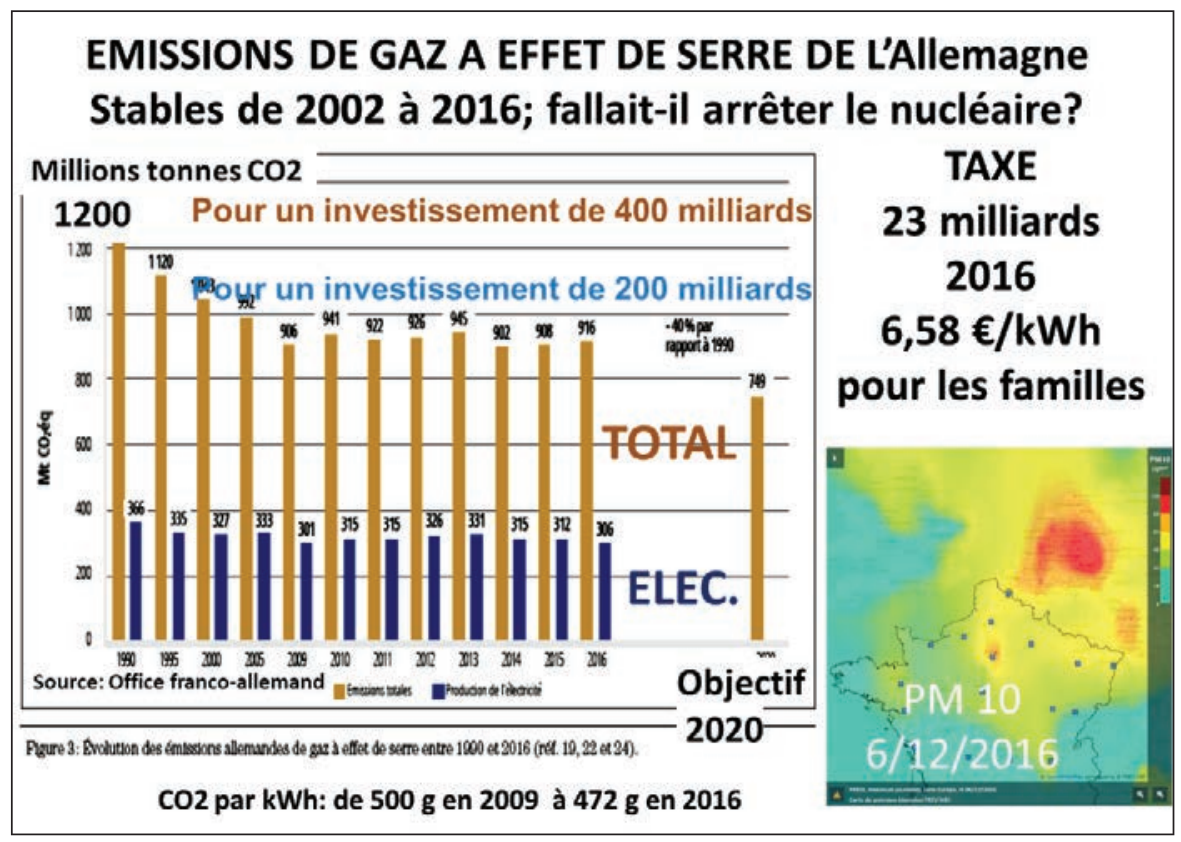

\begin{tabular}{|l|c|c|}
\hline \multicolumn{1}{c|}{} & énages de talle & Industries de taile \\
\hline Danemark & 0,3088 & 0,0602 \\
Allemagne & 0,2969 & 0,0788 \\
Estonie & 0,1208 & 0,0737 \\
Irlande & 0,2306 & 0,1198 \\
Erèce & 0,1760 & 0,0923 \\
France & 0,2185 & 0,1051 \\
Croatie & 0,1685 & 0,0714 \\
\hline
\end{tabular}

Prix comparés de l'électricité (en euros/kWh) en Europe en 2016

Les taxes résultant du soutien aux énergies renouvelables sont en 2016 de 23 milliards d'euros (EEG), représentent depuis l'origine 150 milliards d'euros, et pourraient atteindre un total de 520 milliards d'euros en 2025. 
